Pacific Journal of Mathematics

SOME CLOSURE PROPERTIES FOR TORSION CLASSES OF Bar J. GA 


\title{
SOME CLOSURE PROPERTIES FOR TORSION CLASSES. OF ABELIAN GROUPS
}

\author{
B. J. GARDNER
}

In an earlier paper (B. J. Gardner, Pacific J. Math., 33 (1970), 109-116) the torsion classes of abelian groups which are closed under pure subgroups were characterized, and $\S \S 3-6$ of the present paper are devoted to generalizations of results appearing there. If $\mathscr{C}$ is a homomorphically closed class of objects in an abelian category, a subobject $A$ of an object $B$ is called $\mathscr{C}$-pure if it is a direct summand of every intermediate subobject $X$ for which $X / A \in \mathscr{C}$. (This terminology is due to C. P. Walker). In particular, $\mathscr{C}$ may be a torsion class. The following question is investigated: If $\mathscr{T}$ and $\mathscr{U}$ are torsion classes of abelian groups, when is $\mathscr{T}$ closed under $\mathscr{C}$-pure subgroups? Although ordinary purity is not $\mathscr{U}$-purity for any torsion class $\mathscr{U}$, a torsion class $\mathscr{T}$ is closed under pure subgroups if and only if it is closed under $\mathscr{T}_{0}$-pure subgroups, where $\mathscr{T}_{0}$ is the class of all torsion groups.

In $\S 5$, for an arbitrary torsion theory $(\mathscr{L}, \mathscr{G})$ a rank function ( $\mathscr{U}$-rank) is defined for nonzero groups in $\mathscr{G}$. It is: shown that every torsion class closed under $\mathscr{C}$-pure subgroups. is determined by its intersection with $\mathscr{C}$ and the groups of $\mathscr{U}$-rank 1 it contains. When $\mathscr{C}=\mathscr{T}_{0}$, the groups with $\mathscr{C}^{-}$ rank 1 are the rational groups, so the earlier results for ordinary purity suggest that in general some refinement of the representation should be possible.

A further special case of the general problem is also solved: Let $X$ and $Y$ be rational groups, $T(X), T(Y)$ the smallest torsion classes containing them. If $X$ is a subring of the rationals then $T(X)$ is always closed under $T(Y)$-pure subgroups; if not, the condition is satisfied if and only if $X$ has a greater type than $Y$.

$\S 7$ is devoted to proving the following result: A torsion class is closed under countable direct products, i.e. direct products of countable sets of groups, if and only if it is determined by torsion-free groups.

1. Preliminaries. The basic ideas on torsion classes and theories are contained in Dickson's papers [2], [3], [4]. Every class $\mathscr{C}$ or object $A$ is contained in a smallest torsion class (denoted $T(\mathscr{C})$ or $T(A)$ respectively). This torsion class is said to be determined by the class or object in question.

We shall use the notation of [14] for subfunctors of the identity; thus such a functor $r$ is called a radical if $r(A / r(A))=0$ for every 
$A$ and an idempotent radical if in addition $r(r(A))=r(A)$ for every $A$. Each torsion class $\mathscr{T}$ is associated with an idempotent radical $r$ which assigns to each object its largest subobject from $\mathscr{T}$. Conversely the class of objects fixed by an idempotent radical is a torsion class. The assertion in [3] that a subfunctor of the identity is an idempotent radical if and only if its class of fixed objects is a torsion class is false - the functor need only be a radical, e.g. if $r(G)=$ $\cap n G, n=1,2,3, \cdots$ for every abelian group $G$, then $r(G)=G$ if and only if $G$ is divisible, but $r$ is not idempotent.

The notion of generalized purity we shall be using was introduced in [17].

2. Notation.

$\mathscr{A} \mathscr{B}$
$Z$
$Q$
$Q(p)$
$Q(P)$

category of abelian groups

group of integers

group of rational numbers

group $\left\{m / p^{n} \mid m, n \in Z\right\}$ where $p$ is a prime

group $\{m / n \mid m \in Z, n \in Z$ with prime factors in $P\}$

where $P$ is a set of primes

$I(p) \quad$ group (or ring) of $p$-adic integers

$Z(n) \quad$ cyclic group of order $n$

$Z\left(p^{\infty}\right)$

$\left[x_{\lambda} \mid \lambda \in \Lambda\right]$

quasicyclic $p$-group ( $p$ prime)

$[x]_{*}$

$\tau(x)$

$\tau(X)$

group generated by set $\left\{x_{\lambda} \mid \lambda \in \Lambda\right\}$

smallest pure subgroup containing $x$, where $x$ is an element of a torsion-free group

type of an element $x$ of a torsion-free group

type of a rational group $X$

$\tau\left(h_{1}, h_{2}, \cdots\right)$

type of a height $\left(h_{1}, h_{2}, \cdots\right)$

$A \oplus B, \oplus A_{\lambda}$

ПI $A_{\lambda}$

$\left(a_{\lambda}\right)$

direct $\operatorname{sum}(=$ coproduct $=$ discrete direct sum)

direct product $(=$ product $=$ complete direct sum $)$

element of $\boxplus A_{\lambda}$ or $\Pi A_{\lambda}$

$[A, B]$

$\mathscr{T}_{0}$

group of homomorphism (morphisms) from $A$ to $B$

class of torsion groups

class of torsion-free groups

class of divisible groups

class of $p$-divisible groups, where $p$ is a prime class of $P$-divisible groups, where $P$ is a set of primes.

A torsion class of abelian groups is called a $t$-torsion class if it contains only torsion groups. Throughout the paper, "group" means "abelian group".

For unexplained terms see [7] or [15]. 
3. Products of idempotent radicals. It is shown in [2] that a group $A$ belongs to a torsion class $\mathscr{T}$ if and only if both $A_{t}$ and $A / A_{t}$ do. In this section we shall discuss some generalizations of this result. We shall work in a locally small abelian category $\mathscr{K}$ satisfying the conditions of [3], i.e. if $\left\{K_{\lambda} \mid \lambda \in \Lambda\right\}$ is a set of subobjects of some $K \in \mathscr{K}$, the direct sum $\oplus K_{\lambda}$ and the direct product $\Pi K / K_{\lambda}$ both exist in $\mathscr{K}$. $\mathscr{T}_{1}$ and $\mathscr{T}_{2}$ are torsion classes, $r_{1}$ and $r_{2}$ the associated idempotent radicals.

\section{Proposition 3.1. The statement}

$$
K \in \mathscr{T}_{2} \Longleftrightarrow r_{1}(K), K / r_{1}(K) \in \mathscr{I}_{2}
$$

holds for every $K \in \mathscr{K}$ if and only if $r_{1} r_{2}$ is idempotent.

Proof. If $\left(^{*}\right)$ holds, then for every $K \in \mathscr{K}, r_{2}(K)$ belongs to $\mathscr{T}_{2}$ so $r_{1} r_{2}(K)$ does also, i.e. $r_{2} r_{1} r_{2}(K)=r_{1} r_{2}(K)$, or since $K$ is arbitrary, $r_{2} r_{1} r_{2}=r_{1} r_{2}$, so that $r_{1}\left(r_{2} r_{1} r_{2}\right)=r_{1}\left(r_{1} r_{2}\right)=r_{1} r_{2}$, i.e. $\left(r_{1} r_{2}\right)^{2}=r_{1} r_{2}$. Conversely, let $\left(r_{1} r_{2}\right)^{2}=r_{1} r_{2}$. Then for any $K \in \mathscr{K}$,

$$
r_{1} r_{2}(K)=r_{1} r_{2} r_{1} r_{2}(K) \subseteq r_{2} r_{1} r_{2}(K) \subseteq r_{1} r_{2}(K)
$$

i.e. $r_{1} r_{2}=r_{2} r_{1} r_{2}$. Thus if $K \in \mathscr{T}_{2}$, we have

$$
r_{1}(K)=r_{1} r_{2}(K)=r_{2} r_{1} r_{2}(K)
$$

which is also in $\mathscr{T}_{2}$. Since $\mathscr{T}_{2}$ is closed under homomorphic images and extensions, the proof is complete.

COROLlaRY 3.2. If $r_{1} r_{2}=r_{2} r_{1}$ then

$$
K \in \mathscr{T}_{2} \Longleftrightarrow r_{1}(K), K / r_{1}(K) \in \mathscr{T}_{2}
$$

and

$$
K \in \mathscr{T}_{1} \Longleftrightarrow r_{2}(K), K / r_{2}(K) \in \mathscr{T}_{1} \text {. }
$$

Proof. If $r_{1} r_{2}=r_{2} r_{1}$, then

$$
\left(r_{1} r_{2}\right)^{2}=r_{1}\left(r_{2} r_{1}\right) r_{2}=r_{1}\left(r_{1} r_{2}\right) r_{2}=\left(r_{1} r_{1}\right)\left(r_{2} r_{2}\right)=r_{1} r_{2}
$$

and similarly $\left(r_{2} r_{1}\right)^{2}=r_{2} r_{1}$.

Let $r$ be the idempotent radical for the torsion class $\mathscr{T}_{1} \cap \mathscr{T}_{2}$.

Proposition 3.3. $r_{1} r_{2}$ is idempotent if and only if $r_{1} r_{2}=r$.

Proof. Let $r_{1} r_{2}$ be idempotent with torsion class $\mathscr{K}$. Then for every $K \in \mathscr{T}_{1} \cap \mathscr{T}_{2}$, we have $r_{1} r_{2}(K)=r_{1}(K)=K$, so $\mathscr{T}_{1} \cap \mathscr{T}_{2} \subseteq \mathscr{K}$. 
Since $r_{1} r_{2}=r_{1} r_{2} r_{1} r_{2} \subseteq r_{2} r_{1} r_{2} \subseteq r_{1} r_{2}$, we have $r_{2} r_{1} r_{2}=r_{1} r_{2}$, so $r_{2}(L)=L$

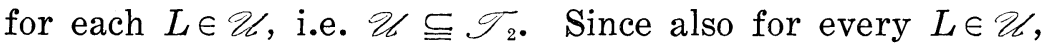

$$
r_{1}(L)=r_{1}\left(r_{1} r_{2}(L)\right)=r_{1}^{2} r_{2}(L)=r_{1} r_{2}(L)=L ，
$$

we have $\mathscr{Z} \subseteq \mathscr{T}_{1}$, so $\mathscr{Q}=\mathscr{T}_{1} \cap \mathscr{T}_{2}$ and $r_{1} r_{2}=r$. The converse is obvious.

Using Corollary 3.2, we obtain

COROLLARY 3.4. $\quad r_{1} r_{2}=r_{2} r_{1}$ if and only if $r_{1} r_{2}$ and $r_{2} r_{1}$ are both idempotent, in which case $r_{1} r_{2}=r_{2} r_{1}=r$.

We now give an example (for $\mathscr{\mathscr { C }}=\mathscr{A} \mathscr{B}$ ) to show that $r_{1} r_{2}$ and $r_{2} r_{1}$ need not be equal. Note that by Corollary 3.4 this is sufficient to show that idempotence is not preserved by products in general.

EXAMPLE 3.5. We consider a group which has been discussed by Erdös [6] and de Groot [11], [12]. Let $\{x, y\}$ be a basis for a 2dimensional rational vector space, and let

$$
G=\left[p^{-n} x, q^{-1} n y, t^{-n}(x+y) \mid n=1,2,3, \cdots\right]
$$

where $p, q$ and $t$ are distinct primes. Let $r_{1}$ and $r_{2}$ be the idempotent radicals for $\mathscr{D}_{p}$ and $T(G)$ respectively. From an examination of the type set of $G$ (see [11] p. 295), it is clear that

$$
r_{1}(G)=\left[p^{-n} x \mid n=1,2,3, \cdots\right] \cong Q(p) .
$$

Let $f$ be any homomorphism from $G$ to $r_{1}(G)$. Since $r_{1}(G)$ has no nonzero elements of infinite $q$-height or $t$-height, we have $f(y)=0=$ $f(x+y)=f(x)+f(y)$. But then $f(x)=0$ and thus $f=0$. Hence $\left[G, r_{1}(G)\right]=0$, so $r_{2} r_{1}(G)=0$. But $r_{1} r_{2}(G) \cong Q(p)$.

4. A simplification of the problem. In this section we shall work in an abelian category $\mathscr{C}$ satisfying the conditions of $\S 3$ and in addition having enough projectives and global dimension 1 . The extra conditions are necessary for some results from [17] which will be used.

Lemma 4.1. An exact sequence

$$
0 \longrightarrow A \longrightarrow B \longrightarrow C \longrightarrow 0
$$

is $\mathscr{C}$-pure, for a torsion class $\mathscr{C}$ if and only if the induced sequence 
$(* *)$

$$
0 \longrightarrow[K, A] \longrightarrow[K, B] \longrightarrow[K, C] \longrightarrow 0
$$

is exact for every $K \in \mathscr{W}$.

Proof. By Theorem 2.7 of [17], $\left(^{*}\right)$ is $\mathscr{C}$-pure if and only if the induced sequence

$$
0 \longrightarrow[L, A] \longrightarrow[L, B] \longrightarrow[L, C] \longrightarrow 0
$$

is exact whenever $L$ is $\mathscr{U}$-pure projective. Theorem 2.6 of [17], the closure properties of $\mathscr{U}$ and our assumption concernig the global dimension of $\mathscr{K}$ jointly imply that the $\mathscr{C}$-pure projectives are the objects of the form $M \oplus K$, where $M$ is projective and $K \in \mathscr{U}$. For such objects, $\left(^{*}\right)$ induces a homomorphism

$$
[M, B] \oplus[K, B] \stackrel{f \oplus g}{\longrightarrow}[M, C] \oplus[K, C]
$$

where $f$ is an epimorphism, so if $\left(^{* *}\right)$ is assumed exact for every $K \in \mathscr{L}, f \oplus g$ is an epimorphism, so $\left(^{*}\right)$ is $\mathscr{U}$-pure. The converse is obvious.

COROLLARY 4.2. Let $s$ be the idempotent radical for $\mathscr{U}$. Then $s(K)$ is $\mathscr{C}$-pure in $K$ for every $K \in \mathscr{K}$.

COROLlaRY 4.3. Let $(\mathscr{U}, \mathscr{G})$ be a torsion theory for $\mathscr{K}$, $K^{\prime} \subseteq K \in \mathscr{G}$. Then $K^{\prime}$ is $\mathscr{C}$-pure if and only if $K / K^{\prime} \in \mathscr{G}$.

The principal result of this section is a generalization of Theorem 3.2 of [9], in which $\mathscr{C}$-purity replaces purity. Proof of this requires

Proposition 4.4. Let $(\mathscr{T}, \mathscr{F})$ and $(\mathscr{U}, \mathscr{G})$ be torsion theories for $\mathscr{K}$, with associated idempotent radicals $r$ and $s$. If $\mathscr{T}$ is closed. under $\mathscr{U}$-pure subobjects, then $s r$ is idempotent.

Proof. Since for any $K \in \mathscr{K}, s(K)$ is a $\mathscr{C}$-pure subobject, in particular $\operatorname{sr}(K)$ is always $\mathscr{C}$-pure in $r(K)$. By assumption on $\mathscr{T}$, therefore, we have $\operatorname{rsr}(K)=\operatorname{sr}(K)$ for each $K$. But then

$$
(s r)^{2}=s(r s r)=s(s r)=s^{2} r=s r .
$$

THEOREM 4.5. Let $(\mathscr{T}, \mathscr{F})$ and $(\mathscr{U}, \mathscr{G})$ be torsion theories for $\mathscr{K}$ with associated idempotent radicals $r, s$ respectively. Then $\mathscr{T}$ is closed under $\mathscr{C}$-pure subobjects if and only if $s r$ is idempotent and $\mathscr{T} \cap \mathscr{G}$ is closed under $\mathscr{L}$-pure subobjects.

Proof. We first show that $\mathscr{T} \cap \mathscr{C}$ is always closed under $\mathscr{C}$-pure 
subobjects. If $K^{\prime}$ is $\mathscr{C}$-pure in $K \in \mathscr{T} \cap \mathscr{W}$, then $K / K^{\prime} \in \mathscr{C}$ and the sequence

$$
0 \longrightarrow K^{\prime} \longrightarrow K \longrightarrow K / K^{\prime} \longrightarrow 0
$$

is split, by definition of $\mathscr{U}$-purity. Hence $K^{\prime} \in \mathscr{T} \cap \mathscr{C}$.

Now suppose $\mathscr{T} \cap \mathscr{G}$ is closed under $\mathscr{Q}$-pure subobjects and $s r$ is idempotent. If $M^{\prime}$ is $\mathscr{C}$-pure in $M \in \mathscr{T}$, we have a commutative diagram

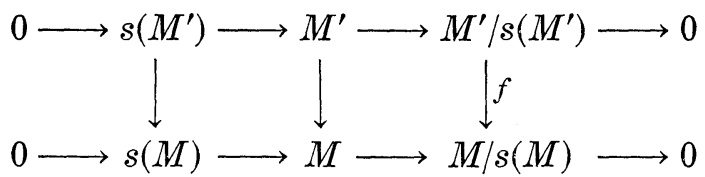

with exact rows.

$f$ is a monomorphism, having kernel $M^{\prime} / s\left(M^{\prime}\right) \cap N$, where $N$ is the kernel of the natural map from $M / s\left(M^{\prime}\right)$ to $M / s(M)$, i.e. $N=$ $s(M) / s\left(M^{\prime}\right)$ and thus

$$
\left(M^{\prime} / s\left(M^{\prime}\right)\right) \cap N=\left(M^{\prime} \cap s(M)\right) / s\left(M^{\prime}\right)=0,
$$

by Corollary 3.5 of [17], which also says that $\left(s(M)+M^{\prime}\right) / M^{\prime}=$ $s\left(M / M^{\prime}\right)$, so

$$
M /\left(s(M)+M^{\prime}\right) \cong\left(M / M^{\prime}\right) /\left(\left(s(M)+M^{\prime}\right) / M^{\prime}\right)=\left(M / M^{\prime}\right) / s\left(M / M^{\prime}\right) \in \mathscr{G} .
$$

Hence the sequence

$$
0 \longrightarrow\left(s(M)+M^{\prime}\right) / s(M) \longrightarrow M / s(M) \longrightarrow M /\left(s(M)+M^{\prime}\right) \longrightarrow 0
$$

is $\mathscr{L}$-pure exact, whence as $M / s(M) \in \mathscr{T} \cap \mathscr{G}$, it follows that

$$
\left(s(M)+M^{\prime}\right) / s(M) \in \mathscr{T} \cap \mathscr{G} \text {. }
$$

But as

$$
M^{\prime} / s\left(M^{\prime}\right)=M^{\prime} /\left(M^{\prime} \cap s(M)\right) \cong\left(M^{\prime}+s(M)\right) / s(M),
$$

this means that $M^{\prime} / s\left(M^{\prime}\right) \in \mathscr{T} \cap \mathscr{G}$.

Also, $s(M) \in \mathscr{T} \cap \mathscr{U}$ (Proposition 3.1). Since $s\left(M^{\prime}\right)$ is $\mathscr{U}$-pure in $M^{\prime}$ and the $\mathscr{C}$-pure short exact sequences form a proper class, $s\left(M^{\prime}\right)$ is $\mathscr{C}$-pure in $M$ and hence in $s(M)$, so $s\left(M^{\prime}\right) \in \mathscr{T} \cap \mathscr{W} . M^{\prime}$ is therefore in $\mathscr{T}$, as both $s\left(M^{\prime}\right)$ and $M^{\prime} / s\left(M^{\prime}\right)$ are, i.e. $\mathscr{T}$ is closed under $\mathscr{C}$-pure subobjects.

By Proposition 4.4 the converse is obvious.

Corollary 4.3 shows that in $\mathscr{F}_{0}$, purity and $\mathscr{T}_{0}$-purity coincide, so as a consequence of Theorem 3.2 of [9] and the last result, we see that in $\mathscr{A} \mathscr{B}$, a torsion class is closed under $\mathscr{T}_{0}$-pure subgroups 
exactly when it is closed under pure subgroups, which raises the question: if $\mathscr{C}$ is homomorphically closed, when is closure of a torsion class under $\mathscr{C}$-pure subobjects equivalent to that for $\mathscr{C}$-pure subobjects for a torsion class $\mathscr{Q}$ ? This question is related to the problem of determining projective closures, for $\mathscr{C}$ and $\mathscr{C}$ satisfy the condition in particular when $\mathscr{C}$-purity coincides with $\mathscr{C}$-purity, i.e. $\mathscr{C}$ and $\mathscr{C}$ have the same projective closure, e.g. if $\mathscr{C}$ is the class of homomorphic images of $Q$ and $\mathscr{U}=\mathscr{D}$ (see [16] or [17]).

5. Groups of generalized rank 1. For the remainder of the paper we shall restrict attention to torsion classes of abelian groups. We note however that the concept we introduce in this section is meaningful for modules over any hereditary ring.

Hereditary torsion classes are determined by the cyclic groups they contain, while torsion classes closed under pure subgroups (or equivalently $\mathscr{T}_{0}$-pure subgroups) are determined by cyclic, quasicyclic and rational groups. We now introduce, for an arbitrary torsion theory $(\mathscr{U}, \mathscr{G})$, a class of groups in $\mathscr{G}$ whose members, together with those of $\mathscr{C}$, determine all torsion classes $\mathscr{T}$ closed under $\mathscr{C}$ pure subgroups. When $\mathscr{C}=\mathscr{T}_{0}$, the groups in question are the rational groups, so the results of [9] indicate that in general a smaller class will suffice. The groups are defined in terms of a rank function associated with $(\mathscr{U}, \mathscr{G})$.

To justify the definition of this rank function the following result is needed:

Proposition 5.1. Let $(\mathscr{L}, \mathscr{G})$ be a torsion theory. If $G \in \mathscr{C}$, then the intersection of any family of $\mathscr{U}$-pure subgroups of $G$ is $\mathscr{Q}$ pure.

Proof. By Corollary 4.3, it suffices to show that $G / \bigcap_{\lambda \in A} G_{\lambda} \in \mathscr{C}$ for any set $\left\{G_{\lambda} \mid \lambda \in \Lambda\right\}$ of $\mathscr{C}$-pure subgroups of $G$. Suppose

$$
\bigcap_{\lambda \in A} G_{\lambda} \subseteq G^{\prime} \subseteq G \quad \text { and } \quad G^{\prime} / \bigcap_{\lambda \in A} G_{\lambda} \in \mathscr{Q}
$$

Then for each $\mu \in \Lambda$, we have a diagram

$$
\begin{aligned}
0 \longrightarrow\left(G^{\prime} \cap G_{\mu}\right) / \bigcap_{\lambda \in A} G_{\lambda} \longrightarrow G^{\prime} / \bigcap_{\lambda \in A} G_{\lambda} \longrightarrow G^{\prime} /\left(G^{\prime} \cap G_{\mu}\right) \longrightarrow 0 \\
\| R \\
0 \longrightarrow\left(G^{\prime}+G_{\mu}\right) / G_{\mu} \longrightarrow G / G_{\mu}
\end{aligned}
$$

with exact rows. $\left(G^{\prime}+G_{\mu}\right) / G_{\mu} \in \mathscr{C}$, but by assumption on $G^{\prime} / \bigcap_{\lambda \in \Lambda} G_{\lambda}$, $G^{\prime} /\left(G^{\prime} \cap G_{u}\right) \in \mathscr{Q}$. 
Hence $G^{\prime} /\left(G^{\prime} \cap G_{\mu}\right)=0$. But this means that $G^{\prime} \subseteq G_{\mu}$ for each $\mu$, so $G^{\prime} / \bigcap_{\lambda \in A} G_{\lambda}=0$, i.e. $G / \bigcap_{\lambda \in A} G_{\lambda} \in \mathscr{G}$.

Every element or subset of a group $G \in \mathscr{G}$ is therefore contained in a smallest $\mathscr{C}$-pure subgroup.

The generalized rank for a torsion theory $(\mathscr{L}, \mathscr{G})$ is introduced in the following definitions.

Definition 5.2. If $\Sigma$ is a subset of a group $G \in \mathscr{G},[\Sigma]_{\sharp}$ denotes: the smallest $\mathscr{U}$-pure subgroup of $G$ containing $\Sigma$. If $\Sigma$ has a single element $x$, we write $[x]_{\varkappa}$ for $[\Sigma]_{\varkappa}$.

Definition 5.3. A nonzero group $G \in \mathscr{G}$ has $\mathscr{C}$-rank $\mathfrak{M}$ if it has a subset $\Sigma$ with $[\Sigma]_{\mathscr{r}}=G$ and $|\Sigma|=\mathfrak{M}$ and there is no set $\Sigma^{r}$ with $\left[\Sigma^{\prime}\right]_{\mathscr{x}}=G$ and $\left|\Sigma^{\prime}\right|<\mathfrak{M}$. We denote this by writing

$$
\mathscr{U}-\operatorname{rank}(G)=\mathfrak{M} \text {. }
$$

$\Sigma$ is then called a $\mathscr{U}$-basis for $G$.

If $(\mathscr{U}, \mathscr{G})=\left(\mathscr{T}_{0}, \mathscr{F}_{0}\right)$ this definition gives the standard rank for nonzero torsion-free groups.

Obviously for every nonzero $x \in G \in \mathscr{G},[x]_{\varkappa}$ has $\mathscr{L}$-rank 1 , so since $G$ is generated by such subgroups, we have

Proposition 5.4. If $(\mathscr{U}, \mathscr{G})$ is a torsion theory then every $G \in \mathscr{G}$ is a homomorphic image of a direct sum of groups in $\mathscr{G}$ with $\mathscr{U}$ rank 1 .

Using Theorem 4.5 and Proposition 5.4 and reasoning as in the proof of Theorem 3.3 of [9], we obtain

THEOREM 5.5. Let $(\mathscr{T}, \mathscr{F})$ and $(\mathscr{U}, \mathscr{G})$ be torsion theories such that $\mathscr{T}$ is closed under $\mathscr{C}$-pure subgroups. Then

$$
\mathscr{T}=T((\mathscr{T} \cap \mathscr{U}) \cup\{G \in \mathscr{T} \cap \mathscr{G} \mid \mathscr{U}-\operatorname{rank}(G)=1\}) .
$$

The groups with $\mathscr{T}_{0}$-rank 1 are the rational groups. Also there is a rank function associated with the trivial torsion theory $(\{0\}$, $\mathscr{A} \mathscr{B})$, with $\{0\}$-rank $(A)=1$ if and only if $A$ is cyclic. Since the hereditary torsion classes are those closed under $\{0\}$-pure subgroups, Theorem 5.5 is a generalization of both Theorem 3.1 of [2] and Theorem 3.3 of [9].

An alternative description of generalized rank is given by 
Proposition 5.6. $G \in \mathscr{G}$ has $\mathscr{L}$-rank $\mathfrak{M}$ if and only if it has a subset $\Sigma$ with $|\Sigma|=\mathfrak{M}$ and $G /[\Sigma] \in \mathscr{Q}$ (here $[\Sigma]$ is the subgroup generated by $\Sigma$ ) and if $\mathfrak{M}$ is th smallest such cardinal number.

Proof. It is clearly sufficient to show that $[\Sigma]_{\varkappa}=G$ if and only if $G /[\Sigma] \in \mathscr{U}$. If $G=[\Sigma]_{\varkappa}$ and $f: G /[\Sigma] \rightarrow H \in \mathscr{G}$ is a homomorphism, then $f g[\Sigma]=0$, where $g: G \rightarrow G /[\Sigma]$ is the natural map. But $f g$ has a $\mathscr{U}$-pure kernel, so $f g=0$, whence $f=0$ and $G /[\Sigma] \in \mathscr{U}$. Conversely, if $G /[\Sigma] \in \mathscr{Q}$, then $G /[\Sigma]_{\mathscr{\varkappa}}$ is both a homomorphic image of $G /[\Sigma]$ and a member of $\mathscr{G}$, so $G /[\Sigma]_{\mathscr{\varkappa}}=0$.

Groups of generalized rank 1 may be decomposable:

EXAMPLE 5.7. $Q(2) \oplus Q(3)$ has $\mathscr{D}_{\{2,3\}}$-rank 1, for if $x$ and $y$ are nonzero elements of $Q(2), Q(3)$, then $Q(2) \oplus Q(3) /[x+y] \cong Q(\{2,3\})$.

A group of $\mathscr{U}$-rank 1 cannot be a direct sum of infinitely many subgroups, since factoring out a cyclic subgroup leaves almost all summands intact. Infinite direct products may have $\mathscr{C}$-rank 1 , however:

EXAMPLE 5.8. The cotorsion completion $\operatorname{Ext}(Q / Z, Z)$ of $Z$, which is isomorphic to $\Pi I(p)$ (all $p$ ) has $\mathscr{D}$-rank 1 .

If a group of generalized rank 1 is decomposable, the corresponding rank of all nonzero summands is also 1 . This follows from

Proposition 5.9. Let $(\mathscr{K}, \mathscr{G})$ be a torsion theory, $G \in \mathscr{G}$, $\mathscr{C}-\operatorname{rank}(G)=1$. Then any nonzero homomorphic image of $G$ which belongs to $\mathscr{G}$ also has $\mathscr{U}$-rank 1.

Proof. Let $\{x\}$ be a $\mathscr{C}$-basis for $G, G^{\prime}$ a proper subgroup of $G$ with $G / G^{\prime} \in \mathscr{G}$. Then $G^{\prime}$ is a $\mathscr{U}$-pure subgroup, so $x \notin G^{\prime}$. Let $\hat{G} / G^{\prime}=\left[x+G^{\prime}\right]_{\mathscr{C}}$. Then $\hat{G} / G^{\prime}$ is $\mathscr{C}$-pure in $G / G^{\prime}$, so since $\mathscr{C}$-purity defines a proper class ([17] Theorem 2.1), $\hat{G}$ is $\mathscr{C}$-pure in $G$ and contains $x$, so that $\hat{G}=G$ and $\left\{x+G^{\prime}\right\}$ is a $\mathscr{C}$-basis for $G / G^{\prime}$.

This result, with Theorem 7.2 below shows that the groups of generalized rank 1 in Examples 5.7 and 5.8 may be replaced by indecomposable groups with the same rank 1 in representations of torsion classes.

The torsion groups with generalized rank 1 are characterized by the following result. 
Proposition 5.10. Let $(\mathscr{C}, \mathscr{G})$ be a torsion theory. A torsion. group in $\mathscr{G}$ has $\mathscr{L}$-rank 1 if and only if it is nonzero cyclic.

Proof. Let $G \in \mathscr{T}_{0} \cap \mathscr{G}$ have a $\mathscr{C}$-basis $\{x\}$. Taking primary decompositions, we have, for every prime $p, G_{p} /[x]_{p} \cong(G /[x])_{p} \in \mathscr{C}$, so it may be assumed that $G$ is a reduced $p$-group and $G /[x]$ is divisible. If $G /[x] \neq 0$, there is induced an exact sequence

$$
0 \longrightarrow[x] \longrightarrow G^{\prime} \longrightarrow Z\left(p^{\infty}\right) \longrightarrow 0
$$

with $G^{\prime} \leqq G$. As $G^{\prime}$ has rank $\leqq 2$ and is reduced, it is bounded and so has no homomorphic image $Z\left(p^{\infty}\right)$. Thus $G=[x]$ is cyclic. The converse is obvious.

We conclude the discussion of the groups of generalized rank 1 by describing the groups with $\mathscr{D}_{p}$-rank 1 , for a prime $p$. As a first. step we prove

LEMmA 5.11. If a group $G$ is such that there is an exact sequence

$$
0 \longrightarrow Z \stackrel{f}{\longrightarrow} G \stackrel{g}{\longrightarrow} Z\left(p^{\infty}\right) \longrightarrow 0
$$

then the p-socle $G[p]$ of $G$ is cyclic (possibly zero).

Proof. Suppose $G[p]$ contains linearly independent elements $x$ and $y$. If there are integers $m, n$ such that $m g(x)+n g(y)=0$, then $m x+n y=f(k)$ for some $k \in Z$. But $p(m x+n y)=0$, so $m x+n y=0$ whence $m x=0=n y$ and $m g(x)=0=n g(y)$, i.e. $g(x), g(y)$ are linearly independent and this is impossible.

Proposition 5.12. Let $G$ have $\mathscr{D}_{p}$-rank 1 and $\mathscr{D}_{p}$-basis $\{x\}$. Then. $G /[x]$ has no summand $Z\left(p^{\infty}\right)$.

Proof. Proposition 5.10 takes care of the torsion case. If $x \in G_{t}$. (the torsion subgroup), then $G_{t} /[x]$ is pure in $G /[x]$ and therefore in $(G /[x])_{t}$, so by Theorem 5.2 of [2] and Proposition 3.1 of [9], $G_{t} /[x] \in \mathscr{D}_{p}$. Proposition 5.10 then implies that $G_{t}$ is cyclic, so $G$ is. split, and this is not possible, since factoring out $[x]$ does not affect a summand complementary to $G_{t}$. Thus $x$ has infinite order.

Suppose $G /[x]$ has a summand $Z\left(p^{\infty}\right)$. Then there is an exact. sequence

$$
0 \longrightarrow[x] \longrightarrow G^{\prime} \longrightarrow Z\left(p^{\infty}\right) \longrightarrow 0
$$

for some subgroup $G^{\prime}$ of $G$ and since $G^{\prime}$ is p-reduced, Lemma 5.11 
says that $G_{t}^{\prime}$ is a (possibly zero) cyclic p-group. Thus $G^{\prime}=G_{t}^{\prime} \oplus H$, where $H$ is torsion-free. $G^{\prime} /(H+[x])$ is a homomorphic image of both $G^{\prime} /[x] \cong Z\left(p^{\infty}\right)$ and the cyclic $G^{\prime} / H \cong G_{t}^{\prime}$. Thus $G^{\prime} /(H+[x])=0$, whence $H / H \cap[x] \cong(H+[x]) /[x]=G^{\prime} /[x] \cong Z\left(p^{\infty}\right)$. Since $H$ is torsionfree and $H \cap[x]$ is cyclic, this means that $H \cong Q(p)$, which is impossible as $G$ is assumed to be $p$-reduced.

CoRollary 5.13. If $\mathscr{D}_{p}$-rank $(G)=1$ then $G$ cannot be mixed.

Proof. If $G$ is mixed, it contains elements of order $p$. Since for every $\mathscr{D}_{p}$-basis $\{x\}, x$ is of infinite order, $G /[x]$ must also have elements of order $p$. But as $G /[x]$ is $p$-divisible, this would require the existence of summands $Z\left(p^{\infty}\right)$.

COROLLARY 5.14. If $\{x\}$ is a $\mathscr{D}_{p}$-basis for $G$ then $[x]$ is a p-pure subgroup of $G$.

Proof. If $G$ is torsion then $G=[x]$, while if $G$ is torsion-free, $G /[x]$ has no elements of order $p$.

Thus every single-element $\mathscr{D}_{p}$-basis generates a $p$-basic subgroup. Clearly also a generator of a cyclic $p$-basic subgroup of a $p$-reduced group determines a $\mathscr{D}_{p}$-basis.

Proposition 5.15. If $\mathscr{D}_{p}$-rank $(G)=1$ and $x \in G$, then $\{x\}$ is a $\mathscr{D}_{p}$-basis if and only if $[x]$ is a p-basic subgroup.

A $p$-reduced torsion group must be a $p$-group, and it is shown in [1] that a torsion-free $p$-reduced group has a cyclic $p$-basic subgroup if and only if it is isomorphic to a $p$-pure subgroup of $I(p)$. These observations, with Proposition 5.15, give a proof of

Theorem 5.16. A group $G$ has $\mathscr{D}_{p}$-rank 1 if and only if it is isomorphic to either a nonzero p-pure subgroup of $I(p)$ or $Z\left(p^{n}\right)$, $n=1,2, \cdots$.

6. An example. Finally we solve the following special case of the generalized pure subgroup closure problem: to find necessary and sufficient conditions on rational groups $X$ and $Y$ for the closure of $T(X)$ under $T(Y)$-pure subgroups.

Our notation for heights, types etc. largely conforms to that of [7], Chapter VII. In particular, $p_{1}, p_{2}, \cdots$ is the natural enumeration of the primes, and in a height $\left(h_{1}, h_{2}, \cdots, h_{n}, \cdots\right), h_{n}$ denotes height at $p_{n}$. 
Theorem 6.1. Let $X, Y$ be rational such that $\tau(X)$ is the type of a height $\left(h_{1}, h_{2}, \cdots, h_{n}, \cdots\right)$ with $0<h_{n}<\infty$ for infinitely many values of $n$. Then $T(X)$ is closed under $T(Y)$-pure subgroups if and only if $\tau(Y) \leqq \tau(X)$.

Proof. Let $(T(Y), \mathscr{F})$ be the torsion theory for $T(Y)$ and let $\left(g_{1}, g_{2}, \cdots, g_{n}, \cdots\right)$ be a height with the same type as $Y$.

If $\tau(Y) \leqq \tau(X)$, then $X \in T(Y)$, so $T(Y)$-pure subgroups are $T(X)$-pure. For groups in $T(X)$, such subgroups are direct summands, and so belong to $T(X)$ themselves.

For the converse we need to consider two cases:

(i) $\tau(Y) \not \measuredangle \tau\left(h_{1}+1, h_{2}+1, \cdots, h_{n}+1, \cdots\right)$. Let $M=\left\{n \mid h_{n}=\infty\right\}$. Let $\left(k_{1}, k_{2}, \cdots, k_{n}, \cdots\right)$ be the subsequence of positive finite terms of $\left(h_{1}, h_{2}, \cdots, h_{n} \cdots\right)$ and re-label the associated primes as $q_{1}, q_{2}, \cdots$. Let $\{x, y\}$ be a basis for a 2-dimensional rational vector space and

$$
G=\left[p^{-n} x, p^{-n} y, q_{n}^{-k_{n}} x, q_{n}^{-k_{n}}\left(q_{n}^{-1} x+y\right) \mid p \in M, n=1,2, \cdots\right] .
$$

A routine argument using the linear independence of $x$ and $y$ shows that $x$ has height $\left(h_{1}, h_{2}, \cdots, h_{n} \cdots\right)$ in $G$. Suppose $y$ is divisible by $q_{n}^{k_{n}}$ for some $n$. Since the same is true of $q_{n}^{-1} x+y, x$ has $q_{n}$-height $k_{n}+1$ at least, which is impossible. Thus $\tau(y)<\tau(x)=\tau(X)$ (in $G$ ). Denoting the coset of $y \bmod .[x]_{*}$ by $\bar{y}$, we have

$$
G /[x]_{*}=\left[p^{-n} \bar{y}, q_{n}^{-k_{n}} \bar{y} \mid p \in M, n=1,2, \cdots\right]
$$

so $G /[x]_{*}$ is rational with type $\tau(X)$. From the exact sequence

$$
0 \longrightarrow X \cong[x]_{*} \longrightarrow G \longrightarrow G /[x]_{*} \cong X \longrightarrow 0
$$

it is clear that $G \in T(X)$.

Observing that $[y]_{*} \notin T(X)$, we now show that $[y]_{*}$ is $T(Y)$-pure in $G$. Let $\bar{x}$ denote the coset of $x \bmod$. $[y]_{*}$. Then

$$
G /[y]_{*}=\left[p^{-n} \bar{x}, q_{n}^{-\left(k_{n}+1\right)} \bar{x} \mid p \in M, n=1,2, \cdots\right]
$$

which is rational of type $\leqq \tau\left(h_{1}+1, h_{2}+1, \cdots, h_{n}+1, \cdots\right)$, so $G /[y]_{*} \in \mathscr{F}$ and $[y]_{*}$ is $T(Y)$-pure in $G$.

(ii) $\tau(Y) \leqq \tau\left(h_{1}+1, h_{2}+1, \cdots, h_{n}+1, \cdots\right)$. Let

$$
U=\left\{p_{n} \mid p_{n} Y=Y\right\} \text { and } S=\left\{p_{n} \mid h_{n}<g_{n}\right\} \text {. }
$$

Note that the our assumption concerning $\tau(Y)$ requires that $p X=X$ for all $p \in U, S$ is infinite and $g_{n}$ is finite for each $p_{n} \in S$. Let

$$
V=\left\{p_{n} \mid h_{n} \geqq g_{n} ; g_{n}<\infty\right\} \text {. }
$$

and re-label the entries of $\left(h_{1}, h_{2}, \cdots, h_{n}, \cdots\right)$ as follows: denote the 
primes $p_{n} \in S$ by $s_{1}, s_{2}, \cdots$, their heights by $k_{1}, k_{2}, \cdots$ and denote the primes in $V$ by $v_{1}, v_{2}, \cdots$ with heights $j_{1}, j_{2}, \cdots$. Finally let

$$
\begin{array}{r}
H=\left[p^{-n} x, s_{n}^{-k_{n}} x, v_{n}^{-j_{n}} x, p^{-n} y, s_{2 n-1}^{-k_{2 n-1}} y, v_{n}^{-j_{n}} y, s_{2 n}^{-k_{2 n}}\left(s_{2 n}^{-1} x+y\right) \mid p \in U,\right. \\
n=1,2, \cdots] .
\end{array}
$$

As in case (i), $\tau(y)<\tau(x)=\tau(X),[x]_{*} \cong X \cong H /[x]_{*}$ and $H \in T(X)$. Also,

$$
H /[y]_{*}=\left[p^{-n} \bar{x}, s_{2 n-1}^{-k_{2 n-1}} \bar{x}, s_{2 n}^{-\left(k_{2 n}+1\right)} \bar{x}, v_{n}^{-j} n \bar{x} \mid p \in U, n=1,2, \cdots\right]
$$

which is rational with type $¥ \tau(Y)$, since it has lower height at infinitely many primes, namely $s_{2 n-1}, n=1,2, \cdots$. Hence $[y]_{*}$ is $T(Y)$ pure in $H$, but $[y]_{*} \notin T(X)$.

The group $G$ of case (i) was used in [10]. Only the case $X=$ $Q(P)$ now remains. Here we prove a more general result.

Proposition 6.2. For a set $P$ of primes, let $\mathscr{T}_{P} \cap \mathscr{D}$ be the class of all divisible P-groups. Then $\mathscr{D}_{P}=T(Q(P))$ is closed under $\mathscr{T}_{P} \cap \mathscr{D}$-pure subgroups.

Proof. The idempotent radical associated with $\mathscr{T}_{P} \cap \mathscr{D}$ is easily seen to commute, and therefore have idempotent products, with all others. Thus by Theorem 4.5 we need only consider $P$-divisible groups without direct summands $Z\left(p^{\infty}\right), p \in P$. If in the exact sequence

$$
0 \longrightarrow A^{\prime} \longrightarrow A \longrightarrow A^{\prime \prime} \longrightarrow 0
$$

$A$ and $A^{\prime \prime}$ are such groups (and thus $\left(^{*}\right)$ is $\mathscr{T}_{P} \cap \mathscr{D}$-pure), then $A^{\prime \prime}$ has zero $p$-component for every $p \in P$. But then $A^{\prime}$ is $P$-pure in $A$, so $A^{\prime} \in \mathscr{D}_{P}$.

THEOREM 6.3. $\mathscr{D}_{P}=T(Q(P))$ is closed under $\mathscr{L}$-pure subgroups, for a torsion class $\mathscr{Q}$, if and only if $\mathscr{Q}$ contains $Z\left(p^{\infty}\right)$ for every $p \in P$.

Proof. Since for $\mathscr{U} \supseteqq\left\{Z\left(p^{\infty}\right) \mid p \in P\right\}$, $\mathscr{C}$-pure subgroups are $\mathscr{T}_{P} \cap \mathscr{D}$-pure, "if" follows from Proposition 6.2. Conversely, if $Z\left(p^{\infty}\right) \notin \mathscr{U}$, for some $p \in P$, then $\mathscr{C}$ is a $t$-torsion class, and $Z\left(p^{\infty}\right) \in \mathscr{G}$, where $(\mathscr{K}, \mathscr{G})$ is the torsion theory of $\mathscr{U}$. The natural exact sequence

$$
0 \longrightarrow Q(P-\{p\}) \longrightarrow Q(P) \longrightarrow Z\left(p^{\infty}\right) \longrightarrow 0
$$

is accordingly $\mathscr{C}$-pure, but $Q(P-\{p\}) \notin \mathscr{D}_{P}$. 
If $\mathscr{Q}$ is not a $t$-torsion class, then $Z\left(p^{\infty}\right) \in \mathscr{C}$ for every prime $p$ ([9], Proposition 2.1). Thus we have

COROLlary 6.4. If a torsion class $\mathscr{Q}$ contains torsion-free groups, (in particular if $\mathscr{U}_{6}=T(Y)$ for some rational $Y$ ), then $\mathscr{D}_{P}$ is closed under $\mathscr{C}$-pure subgroups.

7. Torsion classes closed under countable direct products. We now turn our attention to closure under direct products. The following result will be needed.

LEMMA 7.1. Let $\mathscr{T}$ be a torsion class containing a torsion-free group $A$ which is not $p$-divisible, for some prime $p$. Then $I(p) \in \mathscr{T}$.

Proof. $[A, I(p)] \neq 0([5]$ p. 52) so let $f: A \rightarrow I(p)$ be nonzero and consider $B=I(p) / I m(f) . \quad B / B_{t}$ as a torsion-free proper homomorphic image of $I(p)$ is divisible, (see [5]) and so belongs to $\mathscr{T} . T(I(p)$ ) contains $B$ and therefore $B_{t}$, whence $B_{q}$ is divisible for all primes $q \neq p$. Since in addition $B_{p}$ belongs to $T(A) \subseteq \mathscr{T}$ ([2], Lemma 5.1), $\mathscr{T}$ contains $B_{t}$ and therefore $B$. Since also $\operatorname{Im}(f)$ belongs to $\mathscr{T}$, so does $I(p)$.

The principal result of this section is

THEOREM 7.2. A torsion class $\mathscr{T}$ is closed under countable direct products if and only if it is determined by torsion-free groups.

Most of the proof of Theorem 7.2 is contained in the proofs of the next two results.

Proposition 7.3. Let $A_{n}, n=1,2,3, \cdots$ be torsion-free groups. Then

$$
T\left(\left\{A_{n} \mid n=1,2,3, \cdots\right\}\right)=T\left(\bigoplus_{n=1}^{\infty} A_{n}\right)=T\left(\prod_{n=1}^{\infty} A_{n}\right) .
$$

Proof. The first equality obviously holds; since also $A_{m} \in T\left(\Pi A_{n}\right)$ for each $m$, we have $T\left(\oplus A_{n}\right) \subseteq T\left(\Pi A_{n}\right)$.

Let $f: \Pi A_{n} \rightarrow Y$ be a nonzero epimorphism. If $Y_{p} \neq 0$ for some prime $p$, then if $Y_{p}$ is reduced, we have $p \prod A_{n} \neq \Pi A_{n}$ so $p A_{m} \neq A_{m}$, for some $m$, and thus $Y_{p} \in T\left(A_{m}\right) \subseteq \mathscr{T}$, while if $Y_{p}$ is not reduced, then $\left[A_{n}, Y_{p}\right] \neq 0$ for each $n$.

If $Y$ is torsion-free, then either $f\left(A_{m}\right) \neq 0$ for some $m$ or $f\left(\oplus A_{n}\right)=0$, in which case $f$ factorizes as 


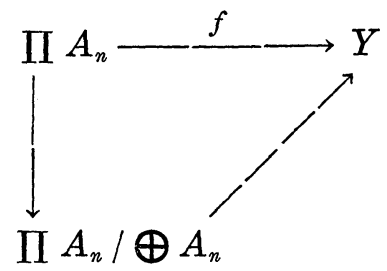

where all maps are epimorphisms. $\Pi A_{n} / \oplus A_{n}$ is algebraically compact (see [13]). Thus $\Pi A_{n} / \oplus A_{n}$ is the direct sum of a divisible group and a (reduced) cotorsion group [8]; so therefore, is $Y$, which being torsion-free is algebraically compact [8]. Thus $Y=D \oplus \Pi R(p)$, where $D$ is divisible and $R(p)$ is inter alia a reduced $I(p)$-module. If $D \neq 0$, then $\left[A_{n}, Y\right] \neq 0$ for each $n$. If $D=0$, let $R(p) \neq 0$. Then $p \Pi A_{n} \neq \Pi A_{n}$ and thus $p A_{m} \neq A_{m}$ for some value of $m$. By Lemma 7.1, $I(p) \in T\left(A_{m}\right)$. Since there is an epimorphism (actually an $I(p)$-epimorphism) from a direct sum of copies of $I(p)$ to $R(p)$, we have $R(p) \in T\left(A_{m}\right)$.

Thus in all cases $\left[A_{m}, Y\right] \neq 0$ for at least one value of $m$, whence $\Pi A_{n}$ belongs to $T\left(\oplus A_{n}\right)$. This completes the proof.

Proposition 7.4. Let $\mathscr{T}=T\left(\left\{A_{\lambda} \mid \lambda \in \Lambda\right\}\right)$, where each $A_{\lambda}$ is torsion-free and let $B_{n}, n=1,2,3, \cdots$ be torsion groups in $\mathscr{T}$. Then. $\mathscr{T}$ contains $\prod_{n=1}^{\infty} B_{n}$.

Proof. Let $f: \Pi B_{n} \rightarrow G$ be a nonzero epimorphism. If for some prime $p, G_{p}$ is nonzero and divisible, then $\left[A_{\lambda}, G_{p}\right] \neq 0$ for each $\lambda \in \Lambda$, while if $G_{p}$ is nonzero but not divisible, then $p \Pi B_{n} \neq \Pi B_{n}$, so $p B_{m} \neq B_{m}$ for some $m$ which means that $p\left(B_{m}\right)_{p} \neq\left(B_{m}\right)_{p}$. Since $\left(B_{m}\right)_{p}$. belongs to $\mathscr{T}$, so do all $p$-groups; in particular $G_{p}$ is in $\mathscr{T}$.

If $G$ is torsion-free, then $f\left(\oplus B_{n}\right)=0$, so $f$ factorizes as

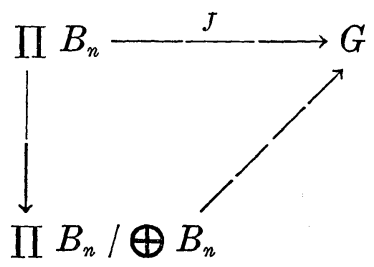

where all maps are epimorphisms. As in Proposition 7.3, $G=$ $D \oplus \Pi R(p), p$ prime, and we need only consider the case where $D=0$. If this is so, and $R(p) \neq 0$, then $p \Pi B_{n} \neq \Pi B_{n}$, and as in the first part of the proof, $\mathscr{T}$ contains all $p$-groups. Hence at. least one $A_{\lambda}$ is not $p$-divisible, so as in Proposition 7.3, $I(p)$ belongs to $\mathscr{T}$ whence $R(p)$ does also. This proves that $\Pi B_{n} \in \mathscr{T}$. 
Proof of Theorem 7.2. If $\mathscr{T}$ is determined by torsion-free groups and if $\left\{A_{n} \mid n=1,2,3, \cdots\right\} \subseteq \mathscr{T}$, then $\left(A_{n}\right)_{t}$ and $A_{n} /\left(A_{n}\right)_{t} \in \mathscr{T}$ for each $n$. By Proposition 7.3, $\Pi A_{n} /\left(A_{n}\right)_{t} \in T\left(\bigoplus A_{n} /\left(A_{n}\right)_{t}\right) \subseteq \mathscr{T}$ and by Proposition 7.4, $\Pi\left(A_{n}\right)_{t} \in \mathscr{T}$, so from the short exact sequence

$$
0 \longrightarrow \Pi\left(A_{n}\right)_{t} \longrightarrow \Pi A_{n} \longrightarrow \Pi A_{n} /\left(A_{n}\right)_{t} \longrightarrow 0
$$

clearly $\Pi A_{n} \in \mathscr{T}$.

Conversely, suppose $\mathscr{T}$ is closed under countable direct products. Clearly $\mathscr{T}$ is not a $t$-torsion class. If it is not determined by torsionfree groups, then for some prime $p, Z(p) \in \mathscr{T}$ but all groups in $\mathscr{T} \cap \mathscr{F}_{0}$ are $p$-divisible. Let $\left[x_{n}\right] \cong Z\left(p^{n}\right), n=1,2, \cdots$. Then $\Pi\left[x_{n}\right] \in \mathscr{T}$, so $\Pi\left[x_{n}\right] /\left(\Pi\left[x_{n}\right]\right)_{t} \in \mathscr{T} \cap \mathscr{F}_{0}$. Suppose

$$
p\left(a_{n} x_{n}\right)-\left(x_{n}\right) \in\left(\Pi\left[x_{n}\right]\right)_{t}, a_{n} \in Z .
$$

Then for some positive $k \in Z, p^{k}\left(p\left(a_{n} x_{n}\right)-\left(x_{n}\right)\right)=0$, so $p^{k}\left(p a_{n}-1\right) x_{n}=0$ for all $n$, i.e. $p^{n} \mid p^{k}\left(p a_{n}-1\right)$. For $n>k$, this means that $p^{n-k} \mid\left(p a_{n}-1\right)$, which is impossible. Thus $\left(x_{n}\right)+\left(\Pi\left[x_{n}\right]\right)_{t}$ has zero $p$-height in $\Pi\left[x_{n}\right] /\left(\Pi\left[x_{n}\right]\right)_{t}$, contradicting the required $p$-divisibility of $\Pi\left[x_{n}\right] /\left(\Pi\left[x_{n}\right]\right)_{t}$.

If $\left\{A_{\lambda} \mid \lambda \in \Lambda\right\} \subseteq \mathscr{D}_{P}$ for any set $P$ of primes, then $\prod_{\lambda \in \Lambda} A_{\lambda} \in \mathscr{D}_{P}$, without any restriction on the size of $\Lambda$. Whether any other torsion classes have this property, or the corresponding one for $|\Lambda|<\mathfrak{M}$, where $\mathfrak{M}>\mathfrak{W}_{0}$, is not known. A related result is

Proposition 7.5. Let $\mathscr{C}$ be a class of slender groups and

$$
\mathscr{T}=\{G \mid[G, C]=0 \text { for all } C \in \mathscr{C}\} \text {. }
$$

Then $\mathscr{T} \cap \mathscr{F}_{0}$ is closed under direct products for which the number of components does not exceed the first cardinal number of nonzero measure.

Proof. Let $\left\{G_{\lambda} \mid \lambda \in \Lambda\right\} \subseteq \mathscr{T} \cap \mathscr{F}_{0}$, where $\Lambda$ has appropriate cardinality. Then for any $C \in \mathscr{C},\left[\oplus G_{k}, C\right]=0$ and consequently for any homomorphism $f: \Pi G_{\lambda} \rightarrow C \in \mathscr{C}, f\left(\oplus G_{\lambda}\right)=0$. By a theorem of Łoś ([7] p. 170), $f=0$, so $\Pi G_{\lambda} \in \mathscr{T}$.

In [2] the problem of classifying all torsion classes (of abelian groups) was reduced to the problem for torsion classes determined by torsion-free groups. Theorem 7.2 therefore says that "all interesting torsion classes are closed under countable direct products". 


\section{REFERENCES}

1. J. W. Armstrong, On the indecomposability of torsion-free abelian groups, Proc. Amer. Math. Soc., 16 (1965), 323-325.

2. S. E. Dickson, On torsion classes of abelian groups, J. Math. Soc. Japan, 17(1965),.. $30-35$.

3. - A torsion theory for abelian categories, Trans. Amer. Math. Soc., 121 (1966), 223-235.

4. - Direct decompositions of radicals, pp. 366-374 of Proceedings of the Conference on Categorical Algebra, La Jolla, 1965. Berlin-Heidelberg-New York: SpringerVerlag 1966.

5. D. W. Dubois, Cohesive groups and p-adic integers, Publ. Math. Debrecen, 12 (1965), 51-58.

6. J. Erdös, On direct decompositions of torsion free abelian groups, Publ. Math. Debrecen, 3 (1954), 281-288.

7. L. Fuchs, Abelian Groups, Budapest: Akadémiai Kiadó, 1958.

8. - Notes on abelian groups II, Acta Math. Acad. Sci. Hungar., 11 (1960), $117-125$.

9. B. J. Gardner, Torsion classes and pure subgroups, Pacific J. Math., 33 (1970), 109-116.

10. - A note on types, Bull. Austral. Math. Soc., 2 (1970), 275-276.

11. J. de Groot, Equivalent abelian groups, Canad. J. Math., 9 (1957), 291-297.

12. Indecomposable abelian groups, Nederl. Akad. Wetensch. Proc. Ser. A., 60 (1957), 137-145.

13. A. Hulanicki, The structure of the factor group of the unrestricted sum by the restricted sum of abelian groups, Bull. Acad. Polon. Sci. Sér. Sci. Math. Astronom. Phys. 10 (1962), 77-80.

14. J.-M. Maranda, Injective structures, Trans. Amer. Math. Soc., 110 (1964), 98-135.

15. B. Mitchell, Theory of Categories, New York-London: Academic Press, 1965.

16. F. Richman, C. Walker and E. A. Walker, Projective classes of abelian groups; pp. 335-343 of Studies on Abelian Groups. Paris: Dunod; Berlin-Heidelberg-New York: Springer-Verlag, 1968.

17. C. P. Walker, Relative homological algebra and abelian groups, Illinois J. Math.,; 10 (1966), 186-209.

Received October 20, 1970.

The University of TASMANia, Hobart, tasmania, Australia 



\section{PACIFIC JOURNAL OF MATHEMATICS}

\section{EDITORS}

H. SAMELSON

Stanford University

Stanford, California 94305

C. R. HobBY

University of Washington

Seattle, Washington 98105
J. DUGUNDJI

Department of Mathematics

University of Southern California

Los Angeles, California 90007

RICHARD ARENS

University of California

Los Angeles, California 90024

\section{ASSOCIATE EDITORS}

E. F. BECKENBACH

B. H. NeumanN

F WoLF

K. YoshidA

\section{SUPPORTING INSTITUTIONS}

UNIVERSITY OF BRITISH COLUMBIA

UNIVERSITY OF SOUTHERN CALIFORNIA

CALIFORNIA INSTITUTE OF TECHNOLOGY

UNIVERSITY OF CALIFORNIA

MONTANA STATE UNIVERSITY

STANFORD UNIVERSITY

UNIVERSITY OF NEVADA

NEW MEXICO STATE UNIVERSITY

OREGON STATE UNIVERSITY

UNIVERSITY OF OREGON

OSAKA UNIVERSITY

UNIVERSITY OF TOKYO

UNIVERSITY OF UTAH

WASHINGTON STATE UNIVERSITY

UNIVERSITY OF WASHINGTON

$\stackrel{*}{*} \stackrel{*}{*} \stackrel{*}{*}{ }^{*}{ }^{*}$ MMEICAN MATHEMATICAL SOCIETY

NAVAL WEAPONS CENTER

Printed in Japan by International Academic Printing Co., Ltd., Tokyo, Japan 


\section{Pacific Journal of Mathematics}

\section{Vol. 42, No. $1 \quad$ January, 1972}

Tage Bai Andersen, On Banach space valued extensions from split faces ........

David Marion Arnold, A duality for quotient divisible abelian groups of finite

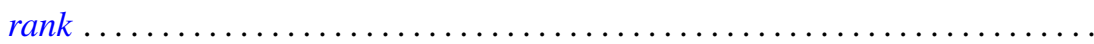

Donald Pollard Ballou, Shock sets for first order nonlinear hyperbolic

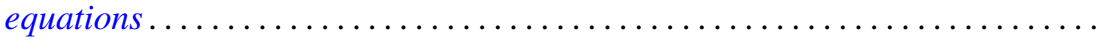

Leon Brown and Lowell J. Hansen, On the range sets of $H^{p}$ functions .........

Alexander Munro Davie and Arne Stray, Interpolation sets for analytic

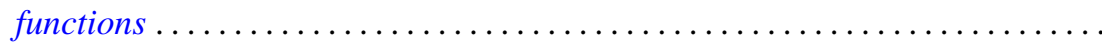

M. G. Deshpande, Structure of right subdirectly irreducible rings. II . . . . . . . . .

Barry J. Gardner, Some closure properties for torsion classes of abelian

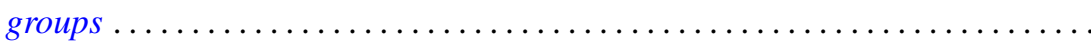

Paul Daniel Hill, Primary groups whose subgroups of smaller cardinality are

direct sums of cyclic groups . . . . . . . . . . . . . . . . . . .

Richard Allan Holzsager, When certain natural maps are equivalences .........

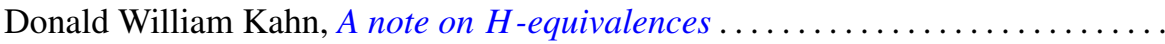

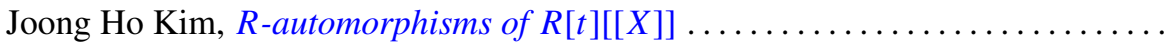

Shin'ichi Kinoshita, On elementary ideals of polyhedra in the 3-sphere.........

Andrew T. Kitchen, Watts cohomology and separability...

Vadim Komkov, A technique for the detection of oscillation of second order

ordinary differential equations .

Charles Philip Lanski and Susan Montgomery, Lie structure of prime rings of characteristic 2

Andrew Lenard, Some remarks on large Toeplitz determinants . .

Kathleen B. Levitz, A characterization of general Z.P.I.-rings. II .

Donald A. Lutz, On the reduction of rank of linear differential systems

David G. Mead, Determinantal ideals, identities, and the Wronskian ...

Arunava Mukherjea, A remark on Tonelli's theorem on integration in product

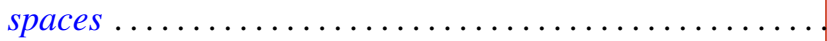

Hyo Chul Myung, A generalization of the prime radical in nonassociative rings.

John Piepenbrink, Rellich densities and an application to unconditionally nonoscillatory elliptic equations.

Michael J. Powers, Lefschetz fixed point theorems for a new class of multi-valued maps .

Aribindi Satyanarayan Rao, On the absolute matrix summability of a Fourier

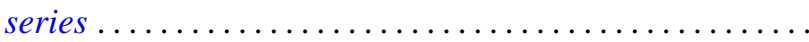

T. S. Ravisankar, On Malcev algebras ......................... 227

William Henry Ruckle, Topologies on sequences spaces . . . . . . . . . . . . . 235

Robert C. Shock, Polynomial rings over finite dimensional rings . . . . . . . . . 251

Richard Tangeman, Strong heredity in radical classes . . . . . . . . . . . . . . 259

B. R. Wenner, Finite-dimensional properties of infinite-dimensional spaces . . . . 267 